\title{
Edwards-Anderson parameter and local Ising-nematicity in FeSe revealed via NMR spectral broadening
}

\author{
Paul Wiecki, ${ }^{1}$ Rui Zhou, ${ }^{2}$ Marc-Henri Julien, ${ }^{2}$ Anna E. Böhmer, ${ }^{1,3}$ and Jörg Schmalian ${ }^{1,4}$ \\ ${ }^{1}$ Institute for Quantum Materials and Technologies, \\ Karlsruhe Institute of Technology (KIT), 76131 Karlsruhe, Germany \\ ${ }^{2}$ Laboratoire National des Champs Magnétiques Intenses - European Magnetic Field Laboratory, \\ UPR3228 Centre National de la Recherche Scientifique, Univ. Grenoble Alpes, \\ Institut National des Sciences Appliquées de Toulouse, Univ. Paul Sabatier, Grenoble, France \\ ${ }^{3}$ Institut für Experimentalphysik IV, Ruhr-Universität Bochum, 44801 Bochum, Germany \\ ${ }^{4}$ Institute for Theory of Condensed Matter, Karlsruhe Institute of Technology (KIT), 76131 Karlsruhe, Germany
}

(Dated: July 21, 2021)

\begin{abstract}
The NMR spectrum of FeSe shows a dramatic broadening on cooling towards the bulk nematic phase at $T_{s}=90 \mathrm{~K}$, due to the formation of a quasi-static, short-range-ordered nematic domain structure. However, a quantitative understanding of the NMR broadening and its relationship to the nematic susceptibility is still lacking. Here, we show that the temperature and pressure dependence of the broadening is in quantitative agreement with the mean-field Edwards-Anderson parameter of an Ising-nematic model in the presence of random-field disorder introduced by nonmagnetic impurities. Furthermore, these results reconcile the interpretation of NMR and Raman spectroscopy data in FeSe under pressure.
\end{abstract}

\section{INTRODUCTION}

The nucleation of local-symmetry-breaking order in nominally symmetry-preserving phases is an increasingly recognized phenomenon in correlated electron systems. Important examples include the formation of antiferromagnetic droplets in $\mathrm{CeCoIn}_{5}$, high- $T_{c}$ cuprates and low dimensional quantum magnets [1, 4] as well as the nucleation of charge density wave order above the phase transition, in $\mathrm{NbSe}_{2}$ [5]7, $\mathrm{ZrTe}_{3}$ [8, 9], cuprates [10] and $\mathrm{Sn} / \mathrm{Ge}(111)-\alpha$ surfaces [11.

In the iron-based superconductors, signatures of $C_{4}$ symmetry breaking and short-range nematic order have often been found well above the bulk nematic phase transition temperature [12 15], especially from local probe measurements using scanning tunneling microscopy (STM) [16] and nuclear magnetic resonance (NMR) [17 24]. More recently, x-ray and neutron pair distribution function (PDF) studies have revealed the locally orthorhombic nature of the tetragonal paramagnetic phase in the ( $\mathrm{Sr}, \mathrm{Na}) \mathrm{Fe}_{2} \mathrm{As}_{2}$ system [25. Similarly, the re-entrant tetragonal antiferromagnetic state in this hole-doped system was also revealed to have short-range orthorhombic correlations [26]. Furthermore, inelastic $\mathrm{x}$-ray scattering has recently produced insights into the spatial correlation length of nematic fluctuations in ironbased superconductors from the wavevector-dependent softening of acoustic phonons $[27+30]$.

While the existence of local nematicity in the tetragonal phase has been rationalized in terms of residual strains in the crystal [31, it is most naturally explained as a consequence of impurities and vacancies that locally break the fourfold rotation symmetry and thus act as random-field impurities for nematicity. Recent theoretical work has addressed the impact of disorder on the emergence of nematicity in iron-based superconductors [32, 33.

FeSe is a unique iron-based superconductor $\left(T_{c}=8.5\right.$ $\mathrm{K})$ which has a bulk nematic phase below $T_{s}=90 \mathrm{~K}$, but no corresponding magnetic phase. Several experiments have revealed a large nematic susceptibility in the high-temperature phase 34 40. Recent NMR measurements 4143 revealed a prominent broadening of the NMR spectrum on approaching $T_{s}$ from above, which is attributed to the formation of locally-nucleated, shortrange-ordered nematic domains. The local orthorhombicity of unstrained FeSe above $T_{s}$ was later confirmed by x-ray and neutron PDF studies [44, 45] and has been used to rationalize properties of the tetragonal phase [46.

Under applied hydrostatic pressure $p$, the temperaturedependent full-width-at-half-maximum (FWHM) of the NMR spectrum follows a universal curve, interrupted only at the nematic transition temperature $T_{s}(p)$. This pressure-independent behavior of the NMR FWHM has been interpreted as evidence that the nematic fluctuations are robust against pressure application, despite the decrease of $T_{s}(p)$ [41, 42. However, this conclusion is in conflict with Raman spectroscopy measurements [47. which show a rapid suppression of nematic fluctuations with increasing pressure.

In order to resolve this discrepancy it is important to establish a quantitative understanding of the nematic broadening of the NMR spectrum and, in particular, its relation to the nematic susceptibility. Here, we find that the broadening of the NMR spectrum due to locallynucleated nematic order is proportional to the EdwardsAnderson parameter of a random-field Ising model at the mean-field level. Within this picture, the pressureindependence of the NMR FWHM is seen to be a consequence of the pressure-independence of random-field de- 
fects. We conclude that the NMR data are consistent with a suppression of nematic coupling with increasing pressure and are not in conflict with the Raman data.

\section{THEORY}

We consider an Ising nematic system characterized by the pseudo-spin variable $\tau_{i}^{z}$ on a lattice with site $i$. Our analysis is independent of the microscopic origin of nematicity and equally applies to systems with spin-induced nematicity [48 51, nematicity due to orbital ordering [52 55], or systems with a Pomeranchuk instability in the $\ell=2$ angular momentum channel 56 58. In a clean system a finite expectation value $\phi \equiv\left\langle\tau^{z}\right\rangle$ corresponds to nematic order.

We allow for random strain fields $\sim h_{i}$ that locally break the four-fold symmetry. To be specific we consider random strain characterized by the distribution function

$$
p_{\sigma}\left(h_{i}\right)=\frac{1}{\sqrt{2 \pi} \sigma} e^{-\frac{h_{i}^{2}}{2 \sigma^{2}}}
$$

The width $\sigma$ is an energy scale that parameterizes the disorder strength. The order parameter is now $\bar{\phi}=\overline{\left\langle\tau^{z}\right\rangle}$, where \langle\rangle is a thermal average and the overbar is an average over disorder configurations. Despite the random strain one still expects a sharp nematic transition above which $\bar{\phi}=0$, at least for three-dimensional systems and not too strong disorder [59, 60. However, for the problem at hand one expects at any finite randomness a finite Edwards-Anderson parameter

$$
q_{E A}=\overline{\phi^{2}}-(\bar{\phi})^{2},
$$

that characterizes the strength of local disorder variations, even if $\phi$ vanishes on the average. Notice that this is different from the behavior in spin glasses where $q_{E A}$ serves, at least within mean field theory, as "order" parameter of the spin glass state 61]. Nevertheless, there are interesting analogies between our approach and the theory of NMR in spin glasses or relaxor ferroelectrics, where the determination of the Edwards-Anderson parameter has played an important role 62 65. In what follows we will first discuss that $q_{E A}$ is directly related to the width of the NMR spectrum. In a second step we present and solve a simple mean-field model that allows for remarkable agreement between theory and experiment.

\section{Connection to NMR spectrum broadening}

We start our analysis with a brief discussion of the effect of random strain on the NMR spectrum. In a homogeneous case, the NMR spectrum of a system where the nucleus under consideration has one unique position in the lattice and occurs only in one isotope configuration can be expressed as

$$
f(\omega)=\frac{1}{N} \sum_{i=1}^{N} \delta\left(\omega-\omega_{0}\right)
$$

where $\omega_{0}$ is the Larmor frequency and $N$ is the number of nuclei in the sample. We assume that a defect-nucleated local order parameter $\phi_{i}\left(h_{i}\right)$ will give rise to a shift of the local NMR resonance frequency according to $\omega_{0} \rightarrow$ $\omega_{0}+\alpha \phi_{i}\left(h_{i}\right)$. By averaging each site over the disorder distribution $p_{\sigma}\left(h_{i}\right)$ one obtains the NMR spectrum as

$$
\overline{f(\omega)}=\frac{1}{N} \int \prod_{j=1}^{N} d h_{j} p_{\sigma}\left(h_{j}\right) \sum_{i=1}^{N} \delta\left(\omega-\omega_{0}-\alpha \phi_{i}\left(h_{i}\right)\right) .
$$

The broadening $\nu$ of the NMR spectrum is given by the second moment of the distribution $\nu^{2}=\int d \omega \overline{f(\omega)}(\omega-$ $\left.\omega_{0}\right)^{2}$. Carrying through the integrations yields

$$
\begin{aligned}
\nu^{2} & =\frac{1}{N} \int \prod_{j=1}^{N} d h_{j} p_{\sigma}\left(h_{j}\right) \sum_{i=1}^{N} \alpha^{2} \phi_{i}^{2}\left(h_{i}\right) \\
& =\alpha^{2} \overline{\phi^{2}}=\alpha^{2} q_{E A} .
\end{aligned}
$$

Therefore, the FWHM of the NMR spectrum is expected to be proportional to the square root of the EdwardsAnderson parameter:

$$
\nu=\alpha q_{E A}^{1 / 2} .
$$

This analysis was performed under the assumption of vanishing averaged order parameter $\bar{\phi}$. At finite $\bar{\phi}$ one easily finds that $\nu^{2}$ is proportional to the expression for $q_{E A}$ in Eq. (2).

\section{Random-Field Ising-nematic model}

In order to get a quantitative understanding of the temperature and strain dependence of the EdwardsAnderson parameter $q_{E A}$ of Eq. (2), we now perform a simple mean-field analysis of the corresponding random field Ising model. While the random-field Ising model is a theoretical problem of formidable complexity, we will confine ourselves to a mean-field analysis. As we will see, this already allows for a rather detailed understanding of the temperature and pressure dependence of the NMR broadening for FeSe. Mean field behavior of the nematic degrees of freedom is expected for clean systems. As was discussed in Ref. [66], long range strain forces drive the statistical mechanics of the system into a mean field regime in the entire temperature window where an appreciable softening of the shear modulus is observed. In systems with random strain, the situation is more subtle [59, 60] and one expects disorder fluctuations 
at long distances and time scales. However on the local length scale of the measurement of the NMR line-width, a mean field analysis is a reasonable starting point. The description of dynamical phenomena, as determined by the NMR relaxation rate, is likely more subtle and may requiring to go beyond the mean field theory.

The Hamiltonian is expressed as

$$
H=-\sum_{i, j} J_{i, j} \tau_{i}^{z} \tau_{j}^{z}-\sum_{i}\left(h_{0}+h_{i}\right) \tau_{i}^{z},
$$

where $h_{0}$ is an external strain that breaks $C_{4}$ symmetry globally, while $h_{i}$ is a random field at each site. In the spirit of the mean field analysis we approximate this model by the infinite range interaction, where $J_{i, j} \rightarrow$ $J / N$. Then mean field theory becomes exact [67. When $h_{i}=0$, we obtain the familiar equation of state for the order parameter $\phi=\left\langle\tau^{z}\right\rangle$ is $\left(\beta \equiv 1 / k_{B} T\right)$

$$
\phi=\tanh \left[\beta\left(2 J \phi+h_{0}\right)\right] .
$$

The nematic transition temperature of the system without disorder follows as $T_{s}^{(0)}=2 J$.

If we now allow for random stress, the corresponding mean-field equation of state for the order parameter becomes

$$
\bar{\phi}=\int d h p_{\sigma}(h) \tanh \frac{2 J \bar{\phi}+h_{0}+h}{k_{B} T},
$$

which is solved self-consistently for $\bar{\phi}$. To find the nematic phase transition temperature $T_{s} / J$ as a function of disorder $\sigma / J$, we take $h_{0}=0$ and expand the hyperbolic tangent while keeping only terms linear in $\bar{\phi}$ to obtain

$$
\bar{\phi}=\frac{2 J \bar{\phi}}{k_{B} T_{s}} C\left(\frac{\sigma}{k_{B} T_{s}}\right),
$$

where the function $C$ is given by

$$
C(t)=\int d x \frac{e^{-\frac{x^{2}}{2}}}{\sqrt{2 \pi}} \tanh ^{2}(x t) .
$$

The transition temperature $T_{s}(\sigma)$ of the system with disorder is determined by the condition

$$
T_{s}=T_{s}^{(0)} C\left(\frac{\sigma}{k_{B} T_{s}}\right) .
$$

Random strain reduces the transition temperature, but the transition itself remains sharp. In distinction, global external strain $h_{0}$ would smear the transition. The nematic transition reaches $T_{s}=0$ when $\sigma / J=\pi / 2$. As we will see, NMR experiments for FeSe suggest that $\sigma$ is significantly smaller than $\pi J / 2$ where disorder destroys nematic order completely.

The nematic susceptibility above $T_{s}$ is given by

$$
\chi_{\mathrm{nem}}(T)=\left.\frac{\partial \bar{\phi}}{\partial h_{0}}\right|_{h_{0}=0}=\frac{C\left(\frac{\sigma}{k_{B} T}\right)}{T-T_{s}^{(0)} C\left(\frac{\sigma}{k_{B} T}\right)} .
$$

As mentioned above, we have to interpret the effective Ising model as the one that includes all allowed couplings, including the ones that are mediated by the lattice. In fact the mean-field treatment of the clean system is possible because we have included this lattice coupling which leads to an effective long-range interaction [66]. Hence, the nematic susceptibility $\chi_{\text {nem }}$ is proportional to the inverse elastic constant $C_{66}$ [50. It diverges at the actual thermodynamic phase transition, not at the lower Curie-Weiss temperature that one deduces from Raman or elasto-resistivity measurements 68 .

Finally, we determine the Edwards-Anderson order parameter. Taking $h_{0}=0$ above $T_{s}$ where $\bar{\phi}=0$, we have

$$
\begin{aligned}
q_{\mathrm{EA}}(T) & =\int d h p_{\sigma}(h) \tanh ^{2} \frac{h}{k_{B} T} \\
& =1-C\left(\frac{\sigma}{k_{B} T}\right) .
\end{aligned}
$$

Obviously, $q_{E A}$ above $T_{s}$ only depends on temperature and the strength of random strain $\sigma$. As we will see below, this explains the "scaling" of the NMR line width as function of pressure. Changing the nematic coupling constant will then not change the $T$ dependence of $q_{E A}$, but merely set the temperature $T_{s}=T_{s}^{(0)}\left(1-q_{\mathrm{EA}}\left(T_{s}\right)\right)$ where it deviates from its high-temperature behavior. Clearly, the finding that $q_{E A}\left(T>T_{s}\right)$ is independent of $J$ is only valid within the mean-field approximation. We can turn this reasoning around and conclude that a temperature dependent NMR line width that is independent of the value of $T_{s}$ strongly supports that the inhomogeneous nematic state above $T_{s}$ is well captured within a mean field treatment.

We also note that the Edwards-Anderson parameter is not proportional to the nematic susceptibility. However, both are related via

$$
\chi_{\mathrm{nem}}(T)=\frac{1-q_{\mathrm{EA}}(T)}{T-T_{s}^{(0)}\left(1-q_{\mathrm{EA}}(T)\right)} .
$$

Thus, in principle it is possible to determine the Edwards-Anderson parameter from the nematic susceptibility. In practice, the available accuracy of data for $\chi_{\text {nem }}(T)$ turns out not to be sufficient to determine $q_{\mathrm{EA}}(T)$. The reason is that $q_{\mathrm{EA}}(T)$ for FeSe turns out to be significantly smaller that unity. This also explains that the $T$-dependence of $q_{\mathrm{EA}}$ does not significantly change the Curie-Weiss behavior of the nematic susceptibility. Thus $\chi_{\text {nem }}$ is not a sensitive indicator of inhomogeneous nematicity while local probes, such as NMR line-width measurements, are able to reveal the existence and temperature dependence of inhomogeneous nematic regions. 
(a)
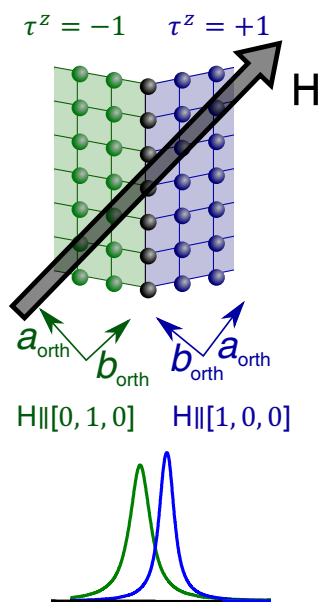

(b)
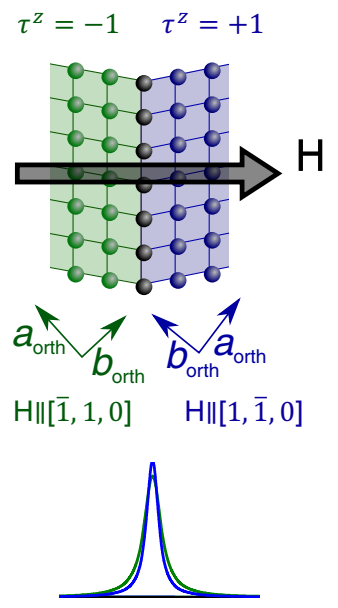

FIG. 1. The Ising variable $\tau^{z}= \pm 1$ breaks the four-fold symmetry in two different ways, resulting in the formation of domains when local nematic order is present. In (a), a magnetic field $H$ is applied such that the nuclei in the two domains experience symmetry-inequivalent field directions. Due to the anisotropy of magnetic susceptibility in the nematic state, the nuclei in the two domains see different local hyperfine fields, resulting in a two-peak NMR spectrum, as illustrated schematically. In contrast, when the field is rotated $45^{\circ}$ as in (b), the nuclei experience symmetry equivalent hyperfine fields, and the NMR spectrum appears as a single peak.

\section{SUMMARY OF NMR RESULTS}

Since ${ }^{77} \mathrm{Se}$ is a $I=1 / 2$ nucleus, there are no quadrupole satellite lines or quadrupole shifts of the spectrum. Therefore, in the high-temperature tetragonal state, the NMR spectrum is a single peak. In the nematic state below $T_{s}$, there are nematic-twin domains. As illustrated in Fig. 11(a), half of the domains experience $H \| a_{O}\left(H \|[100]_{O}\right)$ and the other half experience $H \| b_{O}$ $\left(H \|[010]_{O}\right)$, where $a_{O}>b_{O}$. Due to the anisotropic magnetic susceptibility of the nematic phase [70, 71, the hyperfine field at the nucleus will be different in the two domains, causing the NMR spectrum to split into two peaks in the nematic ordered state. If the field is instead applied along $H \|[110]_{O}$ (Fig. 1(b)), then both types of domain see a symmetry-equivalent magnetic field, and there is only a single NMR peak in the nematic state, even though there are still domains. Recent NMR measurements under mechanical strain have revealed that the higher frequency NMR peak comes from the domains which experience $H \| a_{O}$, while the lower frequency peak comes from the $H \| b_{O}$ domains [71.

In the tetragonal phase, the FWHM of the single NMR peak increases on cooling towards the nematic state. However, this broadening is observed only when $H \|[100]_{O}$ and not when $H \|[110]_{O}$ [41, 42. This observation provides clear evidence that the broadening is related to nematicity and implies the existence of a short-

range nematic domain structure in the tetragonal state of FeSe. Since this effect is observed in the NMR spectrum, the fluctuating nematic domain structure is static on the time scale set by the inverse NMR linewidth $\sim 1 /(1 \mathrm{kHz})$.

\section{COMPARISON OF THEORY AND EXPERIMENT}

For a direct comparison between theory and experiment FeSe offers several clear advantages over other nematic iron-based superconductors. First, FeSe has no bulk magnetic phase at ambient pressure, and thus the pure Ising-nematic model is expected to be relevant in this system. Secondly, the ${ }^{77}$ Se nucleus has $I=1 / 2$ so that no complications arise from nuclear quadrupole couplings. Finally, no dopants are present, which can introduce additional lines into the NMR spectrum [17, 72.

Fig. 2(a) shows the NMR FWHM data for FeSe under pressure. The FWHM increases on cooling towards $T_{s}$. The data at different pressures follow a universal curve, simply interrupted at the appropriate nematic transition temperatures $T_{s}(p)$. Below $T_{s}(p)$ in the nematic state, the FWHM of each of the two NMR peaks is shown separately. The FWHM of the low-frequency NMR peak (open symbols) is greater than that of the high-frequency peak (hatched symbols). The different FWHM of the two NMR peaks in the nematic state of FeSe has been noted in independent measurements and is currently not understood 71].

Within the Ising-nematic model, the experimental data under pressure is expected to correspond to a constant disorder strength $\sigma$ (determined only by the disorder of the particular crystal), but decreasing nematic coupling $J$, starting from a value $J_{0}$ that corresponds to ambient pressure. Figs. 2(b),(c) show the mean-field behavior of the Edwards-Anderson parameter $q_{E A}^{1 / 2}$ and the nematic susceptibility $\chi_{\text {nem }}$ under these assumptions. As expected, the NMR data are well described by $q_{E A}^{1 / 2}$ and not $\chi_{\text {nem }}$ (Eq. 7). The universal FWHM curve followed at all pressures is seen to be the result of the constant $\sigma$. The existence of a universal curve does not mean that the nematic tendency is somehow independent of pressure, as proposed in both NMR papers [41, 42. Rather this reflects the fact that $q_{E A}\left(T>T_{s}\right)$ is independent of $J$ within the mean-field approximation, as discussed above. Therefore, the NMR data are consistent with the Raman data, showing a suppression of nematic fluctuations (Fig. 2(c)) under pressure [47.

The FWHM data in Fig. 2(a) show a deviation from $q_{E A}^{1 / 2}$ at high temperature. This deviation is easily understood. In a usual PM state, the broadening reflects the spatial distribution of $K$ values due to sample inhomogeneity, and the temperature dependence of the broadening typically follows the temperature dependence of the 
(a)

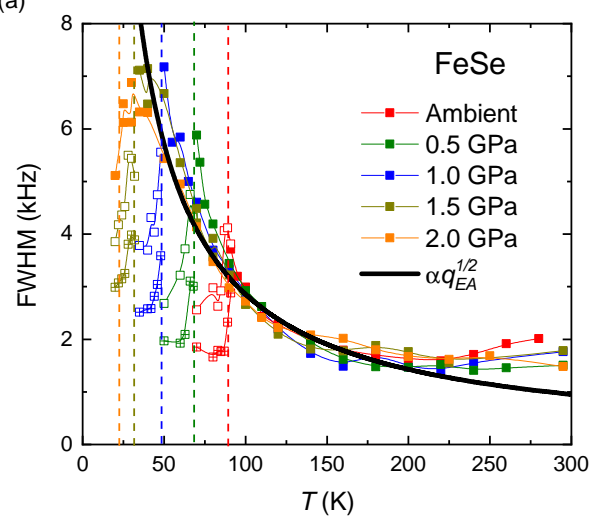

(b)

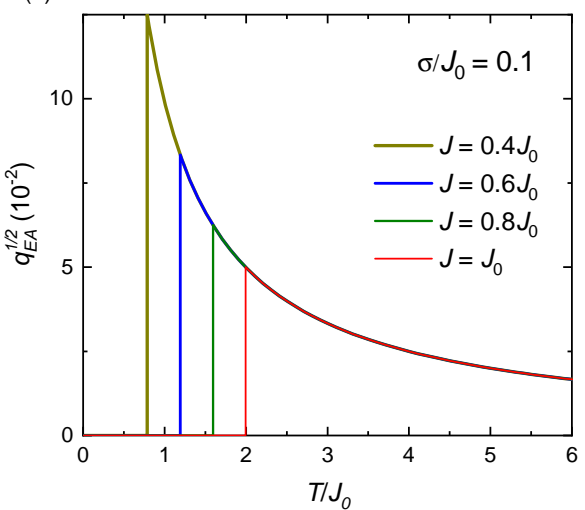

(c)

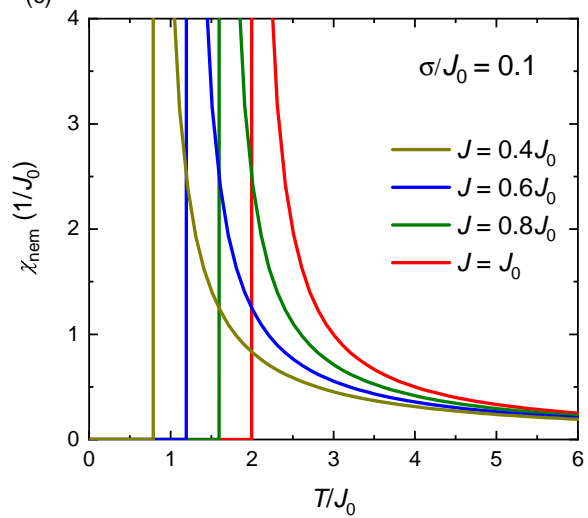

FIG. 2. (a) FWHM of ${ }^{77} \mathrm{Se}$ NMR spectrum in FeSe under indicated hydrostatic pressures 41. Overlaid is the scaled EdwardsAnderson parameter $\alpha q_{E A}^{1 / 2}$ for disorder strength $\sigma / J=0.1$ and $T_{s}=30 \mathrm{~K}$. Vertical dashed lines denote $T_{s}$. Note that at $2.0 \mathrm{GPa}$ $T_{s}$ becomes a joint nematic-magnetic transition 69. Below $T_{s}$, the FWHM of the low-frequency NMR peak (open symbols) is greater than that of the high-frequency peak (hatched symbols). (b) Square root of the Edwards-Anderson parameter $q_{E A}^{1 / 2}$ for decreasing values of nematic coupling $J$ at constant disorder strength $\sigma$. $J_{0}$ corresponds to the strength of the nematic coupling at ambient pressure, while pressure reduces $J$ below $J_{0}$. Notice that $q_{\mathrm{EA}}$ above $T_{s}$ is independent of this nematic interaction $J$. (c) Nematic susceptibility $\chi_{\text {nem }}$ for decreasing values of nematic coupling $J$ at constant disorder strength $\sigma$. The pressure independent behavior of the NMR FWHM in the PM state (a) resembles the Edwards-Anderson parameter $q_{E A}^{1 / 2}(\mathrm{~b})$ and not the nematic susceptibility $\chi_{\text {nem }}(c)$, as anticipated theoretically in Eq. 77).

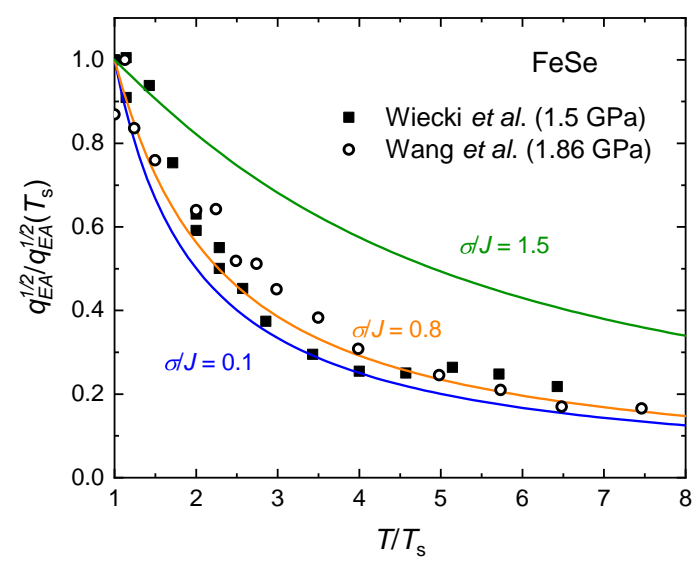

FIG. 3. NMR FWHM in FeSe normalized at $T_{s}$ as a function of $T / T_{s}$ at indicated hydrostatic pressures 41, 42. The data are compared to $q_{E A}^{1 / 2} / q_{E A}^{1 / 2}\left(T_{s}\right)$ calculated in the random-field Ising nematic model at the mean-field level. The only adjustable parameter is the disorder strength $\sigma / J$. For $\sigma / J>$ $\pi / 2$, there is no long-range nematic order.

magnetic susceptibility $\chi_{\text {mag. }}$. In FeSe, both $\chi_{\text {mag }} 38$ and the NMR shift [35, 41, 73] increase with warming, and therefore the NMR spectrum is expected to broaden on warming. At high temperatures $(T \gtrsim 200 K)$, the nematic broadening described by $q_{E A}$ is small and the NMR FWHM is dominated by standard inhomogeneous broadening that increases with increasing temperature. At lower temperatures, the short-range nematic domain structure becomes the dominant source of broadening and the FWHM increases upon decreasing temperature
41.

At $2 \mathrm{GPa}$, the FWHM data appear to level off just above the $T_{s}$. At this pressure, the $T_{s}$ is a joint structural-magnetic transition [69]. Here, the simple Ising-nematic model may no longer be applicable when the ground state is no longer pure nematic.

A more detailed comparison of theory and experiment is shown in Fig. 3, where we plot the FWHM (normalized at $T_{s}$ ) as a function of reduced temperature $T / T_{s}$ for both NMR studies [41, 42. We note that the FWHM at $T_{s}$ differs in the two studies due to different amounts of disorder in the two crystals. Here, we plot the data at high pressure so that $T_{s}$ is reduced and the data can be compared with the Ising-nematic model over the largest temperature range. The only adjustable parameter in the model is the disorder strength $\sigma / J$. Here, the NMR data are compared with $\sigma / J=0.1,0.8$ and 1.5. For $\sigma / J>\pi / 2$, there is no long-range nematic order due to the strong disorder. The NMR data agree well with the theoretical curves at small disorder parameters, as expected.

\section{CONCLUSIONS}

FeSe features a local, orthorhombic nematic order in its high-temperature, nominally tetragonal phase. The observed agreement between the NMR line broadening and the Edwards-Anderson parameter of the disordered, random-field Ising-nematic model implies that this local nematic order is primarily nucleated by crystal defects. The origin of the local orthorhombicity was not explic- 
itly considered in the PDF studies of FeSe [44, 45]. We note that this short-range ordered nematicity in the hightemperature tetragonal state is distinct from the quantum Griffiths phase recently proposed in $\mathrm{Fe}(\mathrm{Se}, \mathrm{S})$ under pressure, where rare but large regions of local nematic droplets undergo quantum fluctuations [74. In this context it is important to keep in mind that in metallic systems such quantum fluctuations are known to be strongly suppressed due to the coupling to the particle-hole continuum [75 77, a behavior specific to Ising degrees of freedoms [78. In fact it is this suppression of quantum fluctuations that is consistent with the effectively classical description of local nematic order of our approach. Finally, our results demonstrate that the nematic fluctuations in FeSe are suppressed by hydrostatic pressure, consistent with Raman studies 47, despite the pressureindependence of the nematic broadening of the NMR lines. This understanding could be further confirmed by elastoresistance measurements inside a pressure cell [79].

We thank B. M. Andersen, S. A. Kivelson, R. M. Fernandes and Y. Furukawa for valuable discussions. We acknowledge support by the Helmholtz Association under Contract No. VH-NG-1242. This work was also supported by the German Research Foundation (DFG) under CRC/TRR 288 (Projects A02 and B01). Work in Grenoble was supported by the Laboratoire d'Excellence LANEF (ANR-10-LABX-51-01) and by the French Agence Nationale de la Recherche (ANR) under reference ANR-19-CE30-0019 (Neptun).

[1] R. R. Urbano, B.-L. Young, N. J. Curro, J. D. Thompson, L. D. Pham, and Z. Fisk, "Interacting antiferromagnetic droplets in quantum critical CeCoIn 5 ," Phys. Rev. Lett. 99, 146402 (2007).

[2] S. Seo, Xin Lu, J-X. Zhu, R. R. Urbano, N. Curro, E. D. Bauer, V. A. Sidorov, L. D. Pham, Tuson Park, Z. Fisk, and J. D. Thompson, "Disorder in quantum critical superconductors," Nature Physics 10, 120-125 (2013).

[3] M.-H. Julien, T. Fehér, M. Horvatić, C. Berthier, O. N. Bakharev, P. Ségransan, G. Collin, and J.-F. Marucco, ${ }^{" 63} \mathrm{Cu}$ NMR evidence for enhanced antiferromagnetic correlations around $\mathrm{Zn}$ impurities in $\mathrm{YBa}_{2} \mathrm{Cu}_{3} \mathrm{O}_{6.7}$," Phys. Rev. Lett. 84, 3422-3425 (2000)

[4] H. Kimura, M. Kofu, Y. Matsumoto, and K. Hirota, "Novel in-gap spin state in zn-doped $\mathrm{La}_{1.85} \mathrm{Sr}_{0.15} \mathrm{CuO}_{4}$," Phys. Rev. Lett. 91, 067002 (2003).

[5] C. Berthier, D. Jerome, and C. Molinie, "NMR study on a $2 \mathrm{~h}-\mathrm{NbSe}_{2}$ single crystal: A microscopic investigation of the charge density waves state," Journal of Physics C: Solid State Physics 11, 797-814 (1978).

[6] C. J. Arguello, S. P. Chockalingam, E. P. Rosenthal, L. Zhao, C. Gutiérrez, J. H. Kang, W. C. Chung, R. M. Fernandes, S. Jia, A. J. Millis, R. J. Cava, and A. N. Pasupathy, "Visualizing the charge density wave transition in $2 \mathrm{H}-\mathrm{NbSe}_{2}$ in real space," Phys. Rev. B 89, 235115 (2014)
[7] U. Chatterjee, J. Zhao, M. Iavarone, R. Di Capua, J. P. Castellan, G. Karapetrov, C. D. Malliakas, M. G. Kanatzidis, H. Claus, J. P. C. Ruff, F. Weber, J. van Wezel, J. C. Campuzano, R. Osborn, M. Randeria, N. Trivedi, M. R. Norman, and S. Rosenkranz, "Emergence of coherence in the charge-density wave state of $2 \mathrm{H}-\mathrm{NbSe}_{2}$," Nature Communications 6, 6313 (2015).

[8] Li Yue, Shangjie Xue, Jiarui Li, Wen Hu, Andi Barbour, Feipeng Zheng, Lichen Wang, Ji Feng, Stuart B. Wilkins, Claudio Mazzoli, Riccardo Comin, and Yuan Li, "Distinction between pristine and disorder-perturbed charge density waves in $\mathrm{ZrTe}_{3}$," Nature Communications 11, 98 (2020)

[9] Limin Liu, Changjiang Zhu, Z. Y. Liu, Hanbin Deng, X. B. Zhou, Yuan Li, Yingkai Sun, Xiong Huang, Shuaishuai Li, Xin Du, Zheng Wang, Tong Guan, Hanqing Mao, Y. Sui, Rui Wu, Jia-Xin Yin, J. G. Cheng, and Shuheng H. Pan, "Thermal dynamics of charge density wave pinning in $\mathrm{ZrTe}_{3}, "$ (2021), arXiv:2105.02640 [cond-mat.str-el].

[10] Tao Wu, Hadrien Mayaffre, Steffen Krämer, Mladen Horvatić, Claude Berthier, W.N. Hardy, Ruixing Liang, D.A. Bonn, and Marc-Henri Julien, "Incipient charge order observed by NMR in the normal state of $\mathrm{YBa}_{2} \mathrm{Cu}_{3} \mathrm{O}_{y}$," Nature Communications 6 (2015), 10.1038/ncomms7438.

[11] Hanno H. Weitering, Joseph M. Carpinelli, Anatoli V. Melechko, Jiandi Zhang, Miroslaw Bartkowiak, and E. Ward Plummer, "Defect-mediated condensation of a charge density wave," Science 285, 2107-2110 (1999), https://science.sciencemag.org/content/285/5436/2107.full.pdf

[12] S. Kasahara, H. J. Shi, K. Hashimoto, S. Tonegawa, Y. Mizukami, T. Shibauchi, K. Sugimoto, T. Fukuda, T. Terashima, Andriy H. Nevidomskyy, and Y. Matsuda, "Electronic nematicity above the structural and superconducting transition in $\mathrm{BaFe}_{2}\left(\mathrm{As}_{1-x} \mathrm{P}_{x}\right)_{2}$," Nature 486, 382-385 (2012)

[13] Ming Yi, Donghui Lu, Jiun-Haw Chu, James G. Analytis, Adam P. Sorini, Alexander F. Kemper, Brian Moritz, Sung-Kwan Mo, Rob G. Moore, Makoto Hashimoto, Wei-Sheng Lee, Zahid Hussain, Thomas P. Devereaux, Ian R. Fisher, and ZhiXun Shen, "Symmetry-breaking orbital anisotropy observed for detwinned $\mathrm{Ba}\left(\mathrm{Fe}_{1-x} \mathrm{Co}_{x}\right)_{2} \mathrm{As}_{2}$ above the spin density wave transition," Proceedings of the National Academy of Sciences 108, 6878-6883 (2011), https://www.pnas.org/content/108/17/6878.full.pdf

[14] Weiyi Wang, Yu Song, Chongde Cao, Kuo-Feng Tseng, Thomas Keller, Yu Li, L. W. Harriger, Wei Tian, Songxue Chi, Rong Yu, Andriy H. Nevidomskyy, and Pengcheng Dai, "Local orthorhombic lattice distortions in the paramagnetic tetragonal phase of superconducting $\mathrm{NaFe}_{1-x} \mathrm{Ni}_{x} \mathrm{As}$," Nature Communications 9 (2018), 10.1038/s41467-018-05529-2

[15] Xiaochen Hong, Federico Caglieris, Rhea Kappenberger, Sabine Wurmehl, Saicharan Aswartham, Francesco Scaravaggi, Piotr Lepucki, Anja U. B. Wolter, Hans-Joachim Grafe, Bernd Büchner, and Christian Hess, "Evolution of the nematic susceptibility in $\mathrm{LaFe}_{1-x} \mathrm{Co}_{x} \mathrm{AsO}$," Phys. Rev. Lett. 125, 067001 (2020)

[16] E. P. Rosenthal, E. F. Andrade, C. J. Arguello, R. M. Fernandes, L. Y. Xing, X. C. Wang, C. Q. Jin, A. J. Millis, and A. N. Pasupathy, "Visualization of electron nematicity and unidirectional antiferroic fluctuations at high temperatures in NaFeAs," Nature Physics 10, 225- 
$232(2014)$

[17] Tetsuya Iye, Marc-Henri Julien, Hadrien Mayaffre, Mladen Horvatić, Claude Berthier, Kenji Ishida, Hiroaki Ikeda, Shigeru Kasahara, Takasada Shibauchi, and Yuji Matsuda, "Emergence of orbital nematicity in the tetragonal phase of $\mathrm{BaFe}_{2}\left(\mathrm{As}_{1-x} \mathrm{P}_{x}\right)_{2}$," Journal of the Physical Society of Japan 84, 043705 (2015).

[18] R. Zhou, L. Y. Xing, X. C. Wang, C. Q. Jin, and Guo-qing Zheng, "Orbital order and spin nematicity in the tetragonal phase of the electron-doped iron pnictides $\mathrm{NaFe}_{1-x} \mathrm{Co}_{x} \mathrm{As}$," Phys. Rev. B 93, 060502 (2016).

[19] Masayuki Toyoda, Yoshiaki Kobayashi, and Masayuki Itoh, "Nematic fluctuations in iron arsenides $\mathrm{NaFeAs}$ and LiFeAs probed by ${ }^{75}$ As NMR," Phys. Rev. B 97, 094515 (2018)

[20] Masayuki Toyoda, Akihiro Ichikawa, Yoshiaki Kobayashi, Masatoshi Sato, and Masayuki Itoh, "In-plane anisotropy of the electric field gradient in $\mathrm{Ba}\left(\mathrm{Fe}_{1-x} \mathrm{Co}_{x}\right)_{2} \mathrm{As}_{2}$ observed by ${ }^{75}$ As NMR," Phys. Rev. B 97, 174507 (2018)

[21] A. P. Dioguardi, M. M. Lawson, B. T. Bush, J. Crocker, K. R. Shirer, D. M. Nisson, T. Kissikov, S. Ran, S. L. Bud'ko, P. C. Canfield, S. Yuan, P. L. Kuhns, A. P. Reyes, H.-J. Grafe, and N. J. Curro, "NMR evidence for inhomogeneous glassy behavior driven by nematic fluctuations in iron arsenide superconductors," Phys. Rev. B 92, $165116(2015)$.

[22] A. P. Dioguardi, T. Kissikov, C. H. Lin, K. R. Shirer, M. M. Lawson, H.-J. Grafe, J.-H. Chu, I. R. Fisher, R. M. Fernandes, and N. J. Curro, "NMR Evidence for Inhomogeneous Nematic Fluctuations in $\mathrm{BaFe}_{2}\left(\mathrm{As}_{1-x} \mathrm{P}_{x}\right)_{2}$," Phys. Rev. Lett. 116, 107202 (2016).

[23] L. Bossoni, P. Carretta, W. P. Halperin, S. Oh, A. Reyes, P. Kuhns, and P. C. Canfield, "Evidence of unconventional low-frequency dynamics in the normal phase of $\mathrm{Ba}\left(\mathrm{Fe}_{1-x} \mathrm{Rh}_{x}\right)_{2} \mathrm{As}_{2}$ iron-based superconductors," Phys. Rev. B 88, 100503 (2013).

[24] L. Bossoni, M. Moroni, M. H. Julien, H. Mayaffre, P. C. Canfield, A. Reyes, W. P. Halperin, and P. Carretta, "Persistence of slow fluctuations in the overdoped regime of $\mathrm{Ba}\left(\mathrm{Fe}_{1-x} \mathrm{Rh}_{x}\right)_{2} \mathrm{As}_{2}$ superconductors," Phys. Rev. B 93, 224517 (2016).

[25] Benjamin A. Frandsen, Keith M. Taddei, Daniel E. Bugaris, Ryan Stadel, Ming Yi, Arani Acharya, Raymond Osborn, Stephan Rosenkranz, Omar Chmaissem, and Robert J. Birgeneau, "Widespread orthorhombic fluctuations in the ( $\mathrm{Sr}, \mathrm{Na}) \mathrm{Fe}_{2} \mathrm{As}_{2}$ family of superconductors," Phys. Rev. B 98, 180505 (2018)

[26] Benjamin A. Frandsen, Keith M. Taddei, Ming Yi, Alex Frano, Zurab Guguchia, Rong Yu, Qimiao Si, Daniel E. Bugaris, Ryan Stadel, Raymond Osborn, Stephan Rosenkranz, Omar Chmaissem, and Robert J. Birgeneau, "Local orthorhombicity in the magnetic $C_{4}$ phase of the hole-doped iron-arsenide superconductor $\mathrm{Sr}_{1-x} \mathrm{Na}_{x} \mathrm{Fe}_{2} \mathrm{As}_{2}$," Phys. Rev. Lett. 119, 187001 (2017)

[27] Shan Wu, Yu Song, Yu He, Alex Frano, Ming Yi, Xiang Chen, Hiroshi Uchiyama, Ahmet Alatas, Ayman H. Said, Liran Wang, Thomas Wolf, Christoph Meingast, and Robert J. Birgeneau, "Short-range nematic fluctuations in $\mathrm{Sr}_{1-x} \mathrm{Na}_{x} \mathrm{Fe}_{2} \mathrm{As}_{2}$ superconductors," Phys. Rev. Lett. 126, 107001 (2021)

[28] F. Weber, D. Parshall, L. Pintschovius, J.-P. Castellan, M. Kauth, M. Merz, Th. Wolf, M. Schütt, J. Schmalian, R. M. Fernandes, and D. Reznik, "Soft phonons reveal the nematic correlation length in $\mathrm{Ba}\left(\mathrm{Fe}_{0.94} \mathrm{Co}_{0.06}\right)_{2} \mathrm{As}_{2}$," Phys. Rev. B 98, 014516 (2018)

[29] A. M. Merritt, F. Weber, J.-P. Castellan, Th. Wolf, D. Ishikawa, A. H. Said, A. Alatas, R. M. Fernandes, A. Q. R. Baron, and D. Reznik, "Nematic correlation length in iron-based superconductors probed by inelastic x-ray scattering," Phys. Rev. Lett. 124, 157001 (2020).

[30] M. Kauth, S. Rosenkranz, A. H. Said, K. M. Taddei, Th. Wolf, and F. Weber, "Soft elastic constants from phonon spectroscopy in hole-doped $\mathrm{Ba}_{1-x}(\mathrm{~K}, \mathrm{Na})_{x} \mathrm{Fe}_{2} \mathrm{As}_{2}$ and $\mathrm{Sr}_{1-x} \mathrm{Na}_{x} \mathrm{Fe}_{2} \mathrm{As}_{2}, "$ Phys. Rev. B 102, 144526 (2020)

[31] S.-H. Baek, D. V. Efremov, J. M. Ok, J. S. Kim, Jeroen van den Brink, and B. Büchner, "Nematicity and inplane anisotropy of superconductivity in $\beta-$ FeSe detected by ${ }^{77}$ Se nuclear magnetic resonance," Phys. Rev. B 93, 180502 (2016)

[32] Laimei Nie, Gilles Tarjus, and Steven Allan Kivelson, "Quenched disorder and vestigial nematicity in the pseudogap regime of the cuprates," Proceedings of the National Academy of Sciences 111, 7980-7985 (2014), https://www.pnas.org/content/111/22/7980.full.pdf

[33] Daniel Steffensen, Panagiotis Kotetes, Indranil Paul, and Brian M. Andersen, "Disorder-induced electronic nematicity," Phys. Rev. B 100, 064521 (2019)

[34] M. D. Watson, T. K. Kim, A. A. Haghighirad, N. R. Davies, A. McCollam, A. Narayanan, S. F. Blake, Y. L. Chen, S. Ghannadzadeh, A. J. Schofield, M. Hoesch, C. Meingast, T. Wolf, and A. I. Coldea, "Emergence of the nematic electronic state in FeSe," Phys. Rev. B 91, 155106 (2015).

[35] A. E. Böhmer, T. Arai, F. Hardy, T. Hattori, T. Iye, T. Wolf, H. v. Löhneysen, K. Ishida, and C. Meingast, "Origin of the tetragonal-to-orthorhombic phase transition in FeSe: A combined thermodynamic and NMR study of nematicity," Phys. Rev. Lett. 114, 027001 (2015).

[36] Chih-Wei Luo, Po Chung Cheng, Shun-Hung Wang, JenChe Chiang, Jiunn-Yuan Lin, Kaung-Hsiung Wu, JenhYih Juang, Dmitry A. Chareev, Olga S. Volkova, and Alexander N. Vasiliev, "Unveiling the hidden nematicity and spin subsystem in FeSe," npj Quantum Materials 2 (2017), 10.1038/s41535-017-0036-5.

[37] Pierre Massat, Donato Farina, Indranil Paul, Sandra Karlsson, Pierre Strobel, Pierre Toulemonde, Marie-Aude Méasson, Maximilien Cazayous, Alain Sacuto, Shigeru Kasahara, Takasada Shibauchi, Yuji Matsuda, and Yann Gallais, "Charge-induced nematicity in FeSe," Proceedings of the National Academy of Sciences 113, 9177-9181 (2016) https://www.pnas.org/content/113/33/9177.full.pdf

[38] Anna E Böhmer and Andreas Kreisel, "Nematicity, magnetism and superconductivity in FeSe," Journal of Physics: Condensed Matter 30, 023001 (2017)

[39] Suguru Hosoi, Kohei Matsuura, Kousuke Ishida, Hao Wang, Yuta Mizukami, Tatsuya Watashige, Shigeru Kasahara, Yuji Matsuda, and Takasada Shibauchi, "Nematic quantum critical point without magnetism in $\mathrm{FeSe}_{1-x} \mathrm{~S}_{x}$ superconductors," Proceedings of the National Academy of Sciences 113, 8139-8143 (2016).

[40] Jack Bartlett, Alexander Steppke, Suguru Hosoi, Hilary Noad, Joonbum Park, Carsten Timm, Takasada Shibauchi, Andrew P. Mackenzie, and Clifford W. Hicks, "The relationship between transport anisotropy and nematicity in FeSe," (2021), arXiv:2102.09212 [cond- 
mat.str-el]

[41] P. Wiecki, M. Nandi, A. E. Böhmer, S. L. Bud'ko, P. C. Canfield, and Y. Furukawa, "NMR evidence for static local nematicity and its cooperative interplay with lowenergy magnetic fluctuations in FeSe under pressure," Phys. Rev. B 96, 180502 (2017)

[42] P. S. Wang, P. Zhou, S. S. Sun, Y. Cui, T. R. Li, Hechang Lei, Ziqiang Wang, and Weiqiang Yu, "Robust short-range-ordered nematicity in FeSe evidenced by high-pressure NMR," Phys. Rev. B 96, 094528 (2017).

[43] J. Li, B. Lei, D. Zhao, L. P. Nie, D. W. Song, L. X. Zheng, S. J. Li, B. L. Kang, X. G. Luo, T. Wu, and X. H. Chen, "Spin-orbital-intertwined nematic state in FeSe," Phys. Rev. X 10, 011034 (2020).

[44] R. J. Koch, T. Konstantinova, M. Abeykoon, A. Wang, C. Petrovic, Y. Zhu, E. S. Bozin, and S. J. L. Billinge, "Room temperature local nematicity in FeSe superconductor," Phys. Rev. B 100, 020501 (2019).

[45] Benjamin A. Frandsen, Qisi Wang, Shan Wu, Jun Zhao, and Robert J. Birgeneau, "Quantitative characterization of short-range orthorhombic fluctuations in FeSe through pair distribution function analysis," Phys. Rev. B 100, 020504 (2019)

[46] Zhi Wang, Xin-Gang Zhao, Robert Koch, Simon J. L. Billinge, and Alex Zunger, "Understanding electronic peculiarities in tetragonal FeSe as local structural symmetry breaking," Phys. Rev. B 102, 235121 (2020).

[47] Pierre Massat, Yundi Quan, Romain Grasset, MarieAude Méasson, Maximilien Cazayous, Alain Sacuto, Sandra Karlsson, Pierre Strobel, Pierre Toulemonde, Zhiping Yin, and Yann Gallais, "Collapse of critical nematic fluctuations in FeSe under pressure," Phys. Rev. Lett. 121, 077001 (2018)

[48] Cenke Xu, Markus Müller, and Subir Sachdev, "Ising and spin orders in the iron-based superconductors," Phys. Rev. B 78, 020501 (2008)

[49] Chen Fang, Hong Yao, Wei-Feng Tsai, JiangPing Hu, and Steven A. Kivelson, "Theory of electron nematic order in LaFeAsO," Phys. Rev. B 77, 224509 (2008)

[50] R. M. Fernandes, L. H. VanBebber, S. Bhattacharya, P. Chandra, V. Keppens, D. Mandrus, M. A. McGuire, B. C. Sales, A. S. Sefat, and J. Schmalian, "Effects of nematic fluctuations on the elastic properties of iron arsenide superconductors," Phys. Rev. Lett. 105, 157003 (2010)

[51] R. M. Fernandes, A. V. Chubukov, and J. Schmalian, "What drives nematic order in iron-based superconductors?" Nature Physics 10, 97-104 (2014).

[52] Frank Krüger, Sanjeev Kumar, Jan Zaanen, and Jeroen van den Brink, "Spin-orbital frustrations and anomalous metallic state in iron-pnictide superconductors," Phys. Rev. B 79, 054504 (2009).

[53] Weicheng Lv, Jiansheng Wu, and Philip Phillips, "Orbital ordering induces structural phase transition and the resistivity anomaly in iron pnictides," Phys. Rev. B 80, 224506 (2009)

[54] C.-C. Chen, J. Maciejko, A. P. Sorini, B. Moritz, R. R. P. Singh, and T. P. Devereaux, "Orbital order and spontaneous orthorhombicity in iron pnictides," Phys. Rev. B 82, 100504 (2010).

[55] Hiroshi Kontani and Seiichiro Onari, "Orbitalfluctuation-mediated superconductivity in iron pnictides: Analysis of the five-orbital Hubbard-Holstein model," Phys. Rev. Lett. 104, 157001 (2010).
[56] Vadim Oganesyan, Steven A. Kivelson, and Eduardo Fradkin, "Quantum theory of a nematic fermi fluid," Phys. Rev. B 64, 195109 (2001).

[57] Hiroyuki Yamase, Vadim Oganesyan, and Walter Metzner, "Mean-field theory for symmetry-breaking Fermi surface deformations on a square lattice," Phys. Rev. B 72, 035114 (2005).

[58] Andrey V. Chubukov, M. Khodas, and Rafael M. Fernandes, "Magnetism, superconductivity, and spontaneous orbital order in iron-based superconductors: Which comes first and why?" Phys. Rev. X 6, 041045 (2016)

[59] T. Nattermann, "Dipolar interaction in random-field systems," Journal of Phys. A: Math. and General 21, 6288 (1988)

[60] H. S. Toh, "Structural phase transitions with random strains," Journal of Phys. A: Math. and General 25, 4767 (1992)

[61] K. H. Fischer and J. A. Hertz, Spin Glasses, Cambridge Studies in Magnetism (Cambridge University Press, 1991).

[62] R. Blinc, "NQR in dipolar and quadrupolar glasses," Zeitschrift für Naturforschung A 45, 313-322 (1990).

[63] Songhua Chen and David C. Ailion, "NMR determination of the Edwards-Anderson order parameter in the deuterated pseudo-spin-glass $\mathrm{Rb}_{1-x}\left(\mathrm{ND}_{4}\right)_{x} \mathrm{D}_{2} \mathrm{PO}_{4}$ : Anisotropy and concentration dependence of the $\mathrm{ND}_{4}^{+}$ deuteron second moment," Phys. Rev. B 42, 5945-5952 (1990)

[64] G. Papantopoulos, G. Papavassiliou, F. Milia, V. H. Schmidt, J. E. Drumheller, N. J. Pinto, R. Blinc, and B. Zalar, " ${ }^{75}$ As nuclear quadrupole resonance in weakly substitutionally disordered $\mathrm{Rb}_{1-x}\left(\mathrm{NH}_{4}\right)_{x} \mathrm{H}_{2} \mathrm{AsO}_{4}$," Phys. Rev. Lett. 73, 276-279 (1994)

[65] R. Blinc, J. Dolinšek, A. Gregorovič, B. Zalar, C. Filipič, Z. Kutnjak, A. Levstik, and R. Pirc, "Local polarization distribution and Edwards-Anderson order parameter of relaxor ferroelectrics," Phys. Rev. Lett. 83, 424-427 (1999)

[66] U. Karahasanovic and J. Schmalian, "Elastic coupling and spin-driven nematicity in iron-based superconductors," Phys. Rev. B 93, 064520 (2016).

[67] T. Schneider and E. Pytte, "Random-field instability of the ferromagnetic state," Phys. Rev. B 15, 1519-1522 (1977)

[68] Yann Gallais and Indranil Paul, "Charge nematicity and electronic Raman scattering in iron-based superconductors," Comptes Rendus Physique 17, 113-139 (2016)

[69] K. Kothapalli, A. E. Böhmer, W. T. Jayasekara, B. G. Ueland, P. Das, A. Sapkota, V. Taufour, Y. Xiao, E. Alp, S. L. Bud'ko, P. C. Canfield, A. Kreyssig, and A. I. Goldman, "Strong cooperative coupling of pressure-induced magnetic order and nematicity in FeSe," Nature Communications 7 (2016), 10.1038/ncomms12728

[70] Mingquan He, Liran Wang, Frédéric Hardy, Liping Xu, Thomas Wolf, Peter Adelmann, and Christoph Meingast, "Evidence for short-range magnetic order in the nematic phase of FeSe from anisotropic in-plane magnetostriction and susceptibility measurements," Phys. Rev. B 97, 104107 (2018)

[71] Rui Zhou, Daniel D. Scherer, Hadrien Mayaffre, Pierre Toulemonde, Mingwei Ma, Yuan Li, Brian M. Andersen, and Marc-Henri Julien, "Singular magnetic anisotropy 
in the nematic phase of FeSe," npj Quantum Materials 5 (2020), 10.1038/s41535-020-00295-1.

[72] F. L. Ning, K. Ahilan, T. Imai, A. S. Sefat, M. A. McGuire, B. C. Sales, D. Mandrus, P. Cheng, B. Shen, and H.-H Wen, "Contrasting spin dynamics between underdoped and overdoped $\mathrm{Ba}\left(\mathrm{Fe}_{1-x} \mathrm{Co}_{x}\right)_{2} \mathrm{As}_{2}$," Phys. Rev. Lett. 104, 037001 (2010)

[73] S-H. Baek, D. V. Efremov, J. M. Ok, J. S. Kim, Jeroen van den Brink, and B. Büchner, "Orbital-driven nematicity in FeSe," Nature Materials 14, 210-214 (2014)

[74] Pascal Reiss, David Graf, Amir A. Haghighirad, Thomas Vojta, and Amalia I. Coldea, "Signatures of a quantum Griffiths phase close to an electronic nematic quantum phase transition," (2021), arXiv:2103.07991 [condmat.str-el]

[75] A. J. Millis, D. K. Morr, and J. Schmalian, "Local defect in metallic quantum critical systems," Phys. Rev. Lett. 87, $167202(2001)$.

[76] A. J. Millis, D. K. Morr, and J. Schmalian, "Quantum Griffiths effects in metallic systems," Phys. Rev. B 66, $174433(2002)$

[77] Thomas Vojta, "Disorder-induced rounding of certain quantum phase transitions," Phys. Rev. Lett. 90, 107202 (2003)

[78] Thomas Vojta and Jörg Schmalian, "Quantum Griffiths effects in itinerant Heisenberg magnets," Phys. Rev. B 72, 045438 (2005).

[79] Elena Gati, Li Xiang, Sergey L. Bud'ko, and Paul C. Canfield, "Measurements of elastoresistance under pressure by combining in-situ tunable quasi-uniaxial stress with hydrostatic pressure," Review of Scientific Instruments 91, 023904 (2020) 